\title{
The Year in Review and What Lies Ahead for First Candle
}

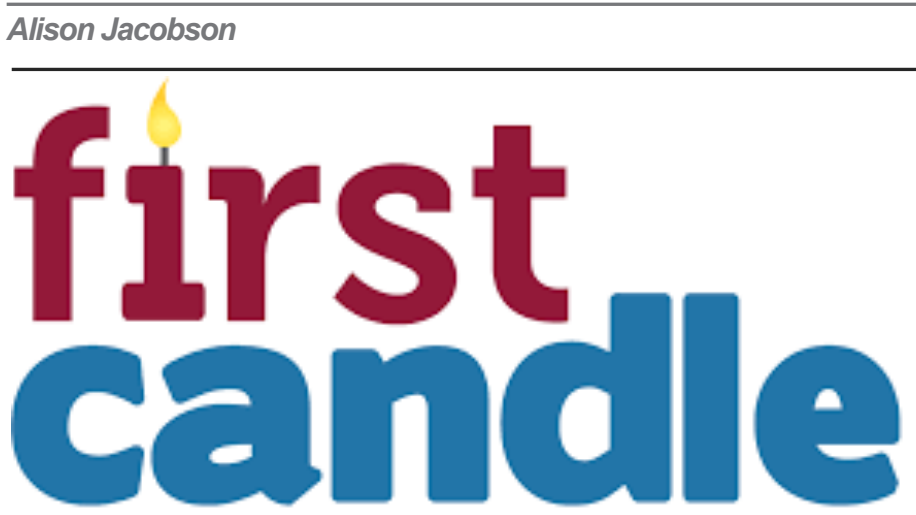

Saving babies. Supporting families.

First Candle's efforts to support families during their most difficult times and provide new answers to help other families avoid the tragedy of the loss of their baby are without parallel.

\section{"This year has been like no other. For many parents who experienced a stillbirth or whose baby died from Sudden Unexpected Infant Death (SUID), First Candle was a much-needed source of comfort and hope when they were forced to remain isolated from friends and family."}

This year has been like no other. For many parents who experienced a stillbirth or whose baby died from Sudden Unexpected Infant Death (SUID), First Candle was a much-needed source of comfort and hope when they were forced to remain isolated from friends and family.

These grief calls made us even more determined to expand our
Straight Talk for Infant Safe Sleep program because we know that practicing the safe sleep guidelines from the American Academy of Pediatrics (AAP) can prevent many of these deaths. While we could not do as many trainings in person, we developed an online curriculum, which was extremely successful. In addition to discussing the safe sleep guidelines, our Straight Talk curriculum continues to include techniques on individual self-reflection to raise awareness of potential bias across racial, socioeconomic and cultural lines and enable those who counsel families on maternal and infant health to assess their own perceptions and effect personal change.

And we have seen how our training makes a difference: $95 \%$ of Straight Talk participants reported gaining improved knowledge about safe sleep guidelines, recognizing their own implicit biases, and strengthening their ability to make them understandable and relevant to the families they work with.

We know that many families still either are not aware of the safe sleep guidelines, choose not to follow them, or have challenges in adopting these practices. We know that there is a great deal of misunderstanding and cultural differences about bed-sharing. Too often, we hear from moms, "I know what's best for my baby, and that includes sleeping with me." We have heard from dads who at times feel sidelined and want to be more involved in supporting their partners in breastfeeding and practicing safe sleep.

Sadly, we know that many SUID deaths are due to Accidental Suffocation and Strangulation in Bed (ASSB), and we are committed to engaging with care providers and families to identify safer sleep strategies. We are working to transform the way families receive information about safe sleep and are supported throughout their pregnancy and beyond when they are overwhelmed and exhausted caring for a new baby.

\section{Community-based Outreach and Advocacy}

In addition to our Straight Talk program, we are creating a grassroots campaign that will utilize community leaders to deliver the new safe sleep guidelines released by the AAP next year. The campaign will reflect the lived experiences of community members and include text-based messages, social media posts, and peer-to-peer interaction. The program will also include "Mamavans" deployed into the community and staffed by public health nurses and safe sleep ambassadors to improve maternal and in-

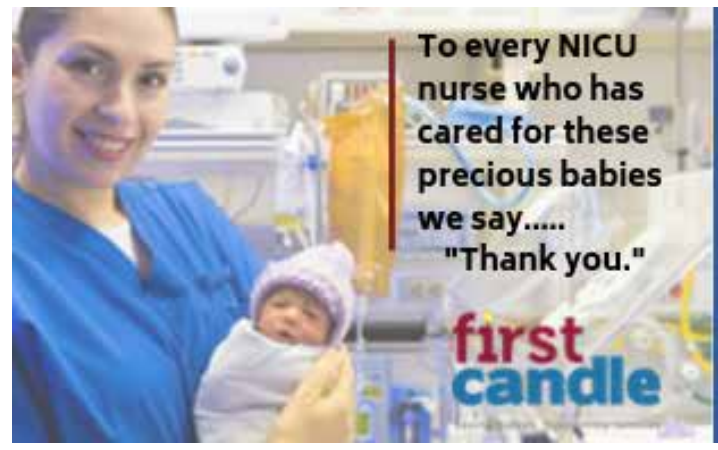

\section{Did you know that premature and low birth weight babies have a $4 \times$ greater risk for SIDS?}

At First Candle we're educating parents, grandparents and caregivers about safer sleep to make sure all babies reach their first birthday. Learn more at firstcandle.org 
fant healthcare access.

At the national level, we are partnering with other national advocacy organizations, including Moms Rising, Black Mamas Matter, and Safe Kids, and supporting legislation such as the MOMS Act that will improve medical coverage postpartum and include doulas under Medicaid.

While this year has certainly been challenging, it has also been rewarding as we continue to expand our reach into communities around the country. We look forward to doing even more next year.

From all of us at First Candle, we wish you a happy, healthy, and safe holiday season.

\section{"At the national level, we are partnering} with other national advocacy organizations, including Moms Rising, Black Mamas Matter, and Safe Kids, and supporting legislation such as the MOMS Act that will improve medical coverage postpartum and include doulas under Medicaid."

\section{About First Candle}

First Candle, based in New Canaan, CT, is a 501c (3) committed to eliminating Sudden Infant Death Syndrome and other sleeprelated infant deaths while providing bereavement support for families who have suffered a loss. Sudden unexpected infant death (SUID), which includes SIDS and accidental suffocation and strangulation in bed (ASSB), remains the leading cause of death for babies one month to one year of age, resulting in 3,600 infant deaths nationwide per year.

Disclosure: The author is the Executive Director and Chief Executive Officer of First Candle, Inc., a Connecticut not for profit 501c3 corporation.

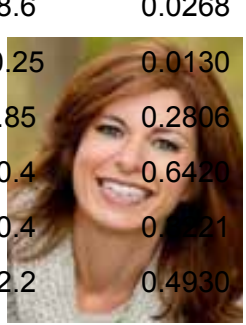

Corresponding Author
Alison Jacobson

Executive Director

Chief Executive Officer

First Candle

49 Locust Avenue, Suite 104

New Canaan, CT 06840

Telephone: 1-203-966-1300

For Grief Support: 1-800-221-7437

www.firstcandle.org

\section{New subscribers are always welcome! NEONATOLOGY TQDAY}

To sign up for free monthly subscription, just click on this box to go directly to our subscription page

NT

The National Urea Cycle Disorders Foundation

Time is precious, just like your patients.
The NUCDF is a non-profit organization dedicated to the identification, treatment and cure of urea cycle disorders. NUCDF is a nationally-recognized resource of information and education for families and healthcare professionals. 